\title{
Identification of the operating parameters for the mechanical system using EMD algorithm
}

\author{
J. Jonak ${ }^{1}$, A. Machrowska ${ }^{1}$, J. Podgórski ${ }^{2}$, and J. Bęc ${ }^{2}$ \\ ${ }^{1}$ Department of Machine Design and Mechatronics, Faculty of Mechanical Engineering, Lublin University of Technology, \\ Poland \\ ${ }^{2}$ Department of Structural Mechanics, Faculty of Civil Engineering and Architecture, Lublin University of Technology, \\ Poland
}

\begin{abstract}
The subject of paper was a research on mechanical system with pneumatic coupling, consisting of a compressor turbine $\left(\mathrm{T}_{\mathrm{I}}\right)$ and of so-called free driving turbine $\left(\mathrm{T}_{\mathrm{II}}\right)$. The purpose of the tests is to determine the applicability of Empirical Mode Decomposition (EMD) algorithm to identify operating parameters of the mechanical system, which is necessary to build a system for structure condition monitoring with use of vibroacoustic method. There has been assumed possibility of easy separation of working frequencies of these components on order to estimate amplitudes generated by them.
\end{abstract}

\section{Introduction}

Identification of the operating parameters for the mechanical systems is crucial for the construction and development of monitoring and diagnosis systems.

Each of the elements of the monitored object is characterized by a number of frequency bands. Knowledge of these bands determines the correctness of the technical condition assessment. One of the most effective methods of appraisal provides the vibration analysis.

Input signal obtained as a result of measurements may constitute a nonlinear and non-stationary data set, resulting in significant limitations in case of application of commonly known analytical methods. The most of the conventional diagnostic methods are based on the assumption of operating parameters stationarity, thus they tend to be unreliable in case of operating parameters changes. As an interesting example of the difficulties associated with the identification of operating parameters, which occur during vibration analysis (performed by conventional techniques) we can indicate fluctuating load conditions [1] or changes in rotational speed of mechanical system components [2].

As a result of numerous literature reports with reference to the possibility of processing strongly nonlinear and non- stationary vibration signals, it was decided to test the EMD algorithm for recorded vibration signals. The object of research was the mechanical system, whose essential components were two turbines $\left(T_{I}\right.$ and $T_{I I}$ turbine) coupled pneumatically.

\section{EMD algorithm}

Adaptive technique Empirical Mode Decomposition (EMD) is a method of time-frequency analysis of multicomponent, nonlinear and non-stationary signals [3].

The basic assumption of this algorithm is to decompose the signal into a finite number of mode oscillations imbedded in the input signal called Intrinsic Mode Functions (IMFs). EMD analyses have found a wide range of applications [3-7].

EMD algorithm is based on the process of sifting the IMFs from given input data set. IMF obtaining is related to local extrema identification. The first phase of this method includes identification of all local extremes. Found local maxima are interpolated by cubic spline in order to build the upper signal envelope. The same procedure is used for the determination of the lower envelope (Figure 1).

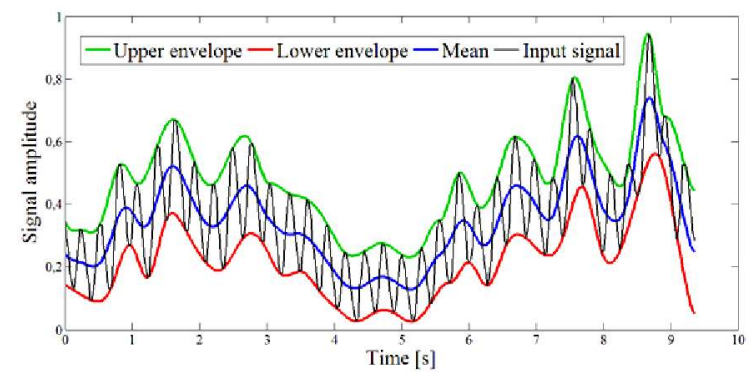

Fig. 1. EMD algorithm: input signal envelopes building 
Subsequently their mean $m_{l}(t)$ is calculated at each point and the difference between input data $x(t)$ and already obtained $m_{l}(t)$ is determined.

$h_{l}(t)=x(t)-m_{l}(t)$

According to Huang [8], function $h_{l}(t)$ is an IMF, when it satisfies two conditions: in the whole data set, the number of extremes and the number of zero crossings must be either equal or differ at most by one, at any point the mean value $m_{l}(t)$ of the envelope defined by the local maxima, and the envelope defined by the local minima is zero.

Sifting procedure is repeated $k$ times for each of the resulting difference $h_{k}(t)$, often referred to prototype mode or shortly: proto-IMF $[9,10]$.

$h_{n}(t)=h_{n-l}(t)-m_{n}(t)$

Process can be stopped when the condition of equality of the zero crossings and extremes number is fulfilled. Then it is determined a residual signal $r_{n}(t)$, described as:

$r_{n}(t)=x(t)-\sum c_{i}(t)$

where $c_{i}(t)$ is a $i$-th Intrinsic Mode Function and $n$ expresses the largest number of mode oscillations possible to obtain.

\section{Tests and results}

\subsection{Signal preprocessing}

There have been recorded time series of acceleration for the analyzed mechanical system. These ones have been numerically elaborated (including integration), in order to conduct the velocity analysis. A typical course, at sampling rate of $12 \mathrm{kHz}$, for the received vibration velocity signal is presented in Figure 2.
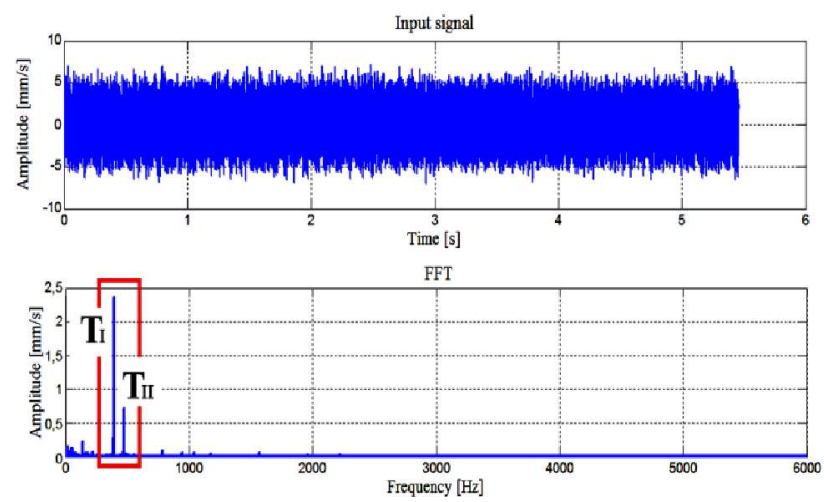

Fig. 2. Time series and spectral analysis of the vibration velocity

\subsection{Results of EMD algorithm}

As the result of using the EMD algorithm, the distribution of the signal at 15 IMFs and the residual signal has been found and shown in Figure 3.
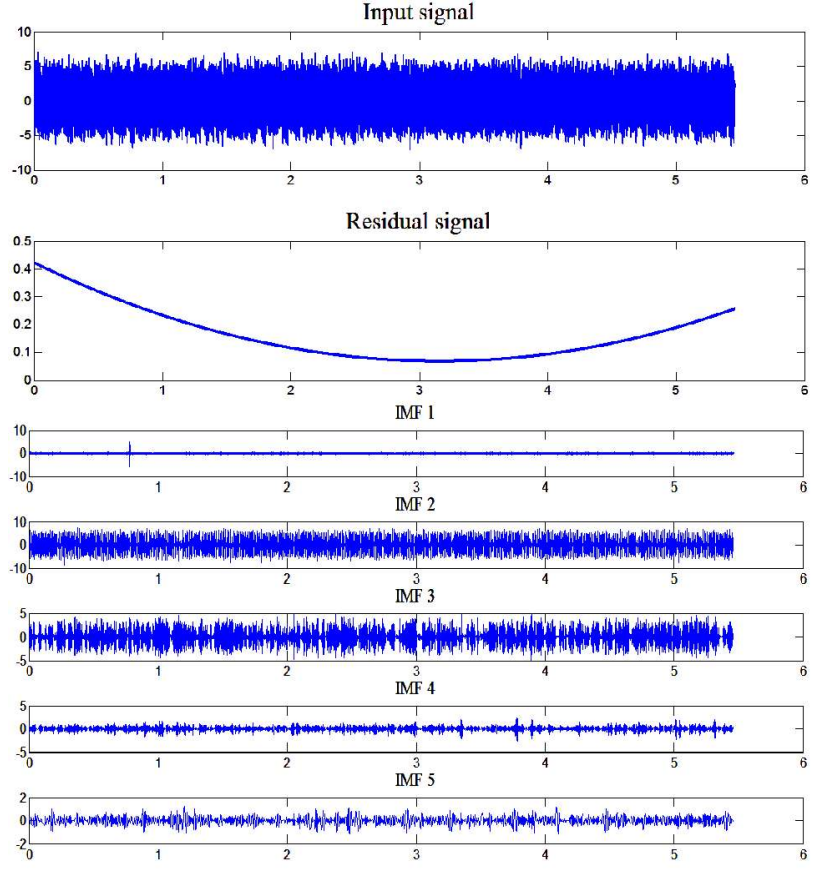

$\mathrm{MMF} 6$

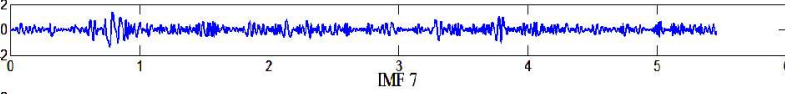

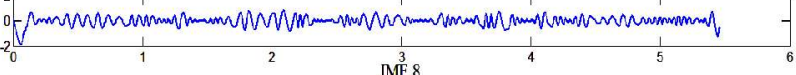

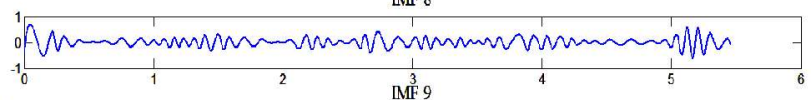

$\underbrace{0.5}_{0.5}$
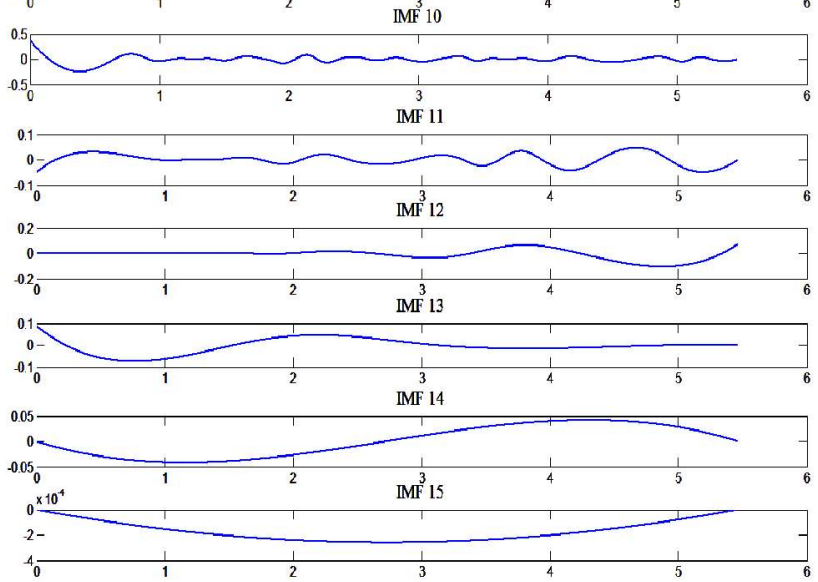

Fig. 3. Distribution of the analyzed signal at $15 \mathrm{IMF}$

In order to verify the results of decomposition for each of the previously extracted IMF functions, FFT (Fast Fourier Transform) analysis was carried out. Research has shown the occurrence of the energy "leakage" effects between IMF 2 and IMF 3 (Figure 4). 

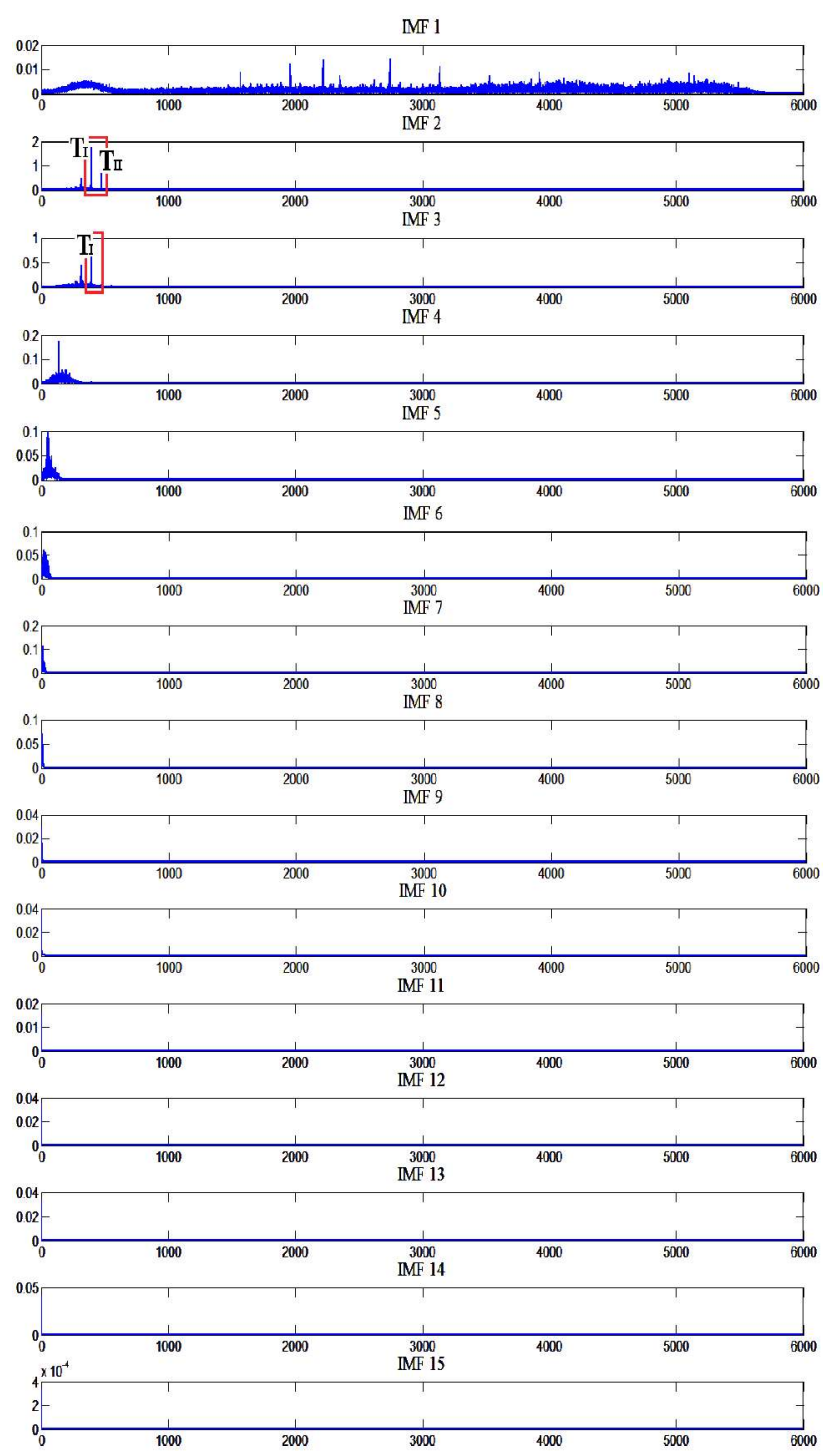

Fig. 4. FFT analysis of the IMF

\subsection{EMD limitations}

Within the framework of EMD algorithm, analytical test has shown that the procedure has some limitations. The first is limitation of the decomposition for the single data channel. The second is bounded resolution arising from the fact of performing the so-called "dyadic" filtration. Another one is observed "energy leakage" phenomenon. As the result, there is no successful separation of the characteristic frequencies for $\mathrm{T}_{\mathrm{I}}$ and $\mathrm{T}_{\mathrm{II}}$ turbines.

\section{Conclusions}

Characteristic for the analyzed mechanical system is the existence of significant angular velocity and amplitude fluctuations. This feature is observed especially for examined object with turbines being the basic components. In such a case, there is a considerable variability of amplitude ratios and rotational frequencies. Equally important is the variability of relative location of characteristic frequencies.
Further analyses showed that the ratio of the $\mathrm{T}_{\mathrm{I}}$ and $\mathrm{T}_{\mathrm{II}}$ frequencies $\left(f T_{I} / f T_{I I}\right)$ was 1,22 with amplitude ratio $\left(A T_{I} / A T_{I I}\right)$ equal to 0,29 . According to the Feldman nomogram (Figure 5) [11], such a parameter set covers an area, where it is impossible to clearly separate signal components.

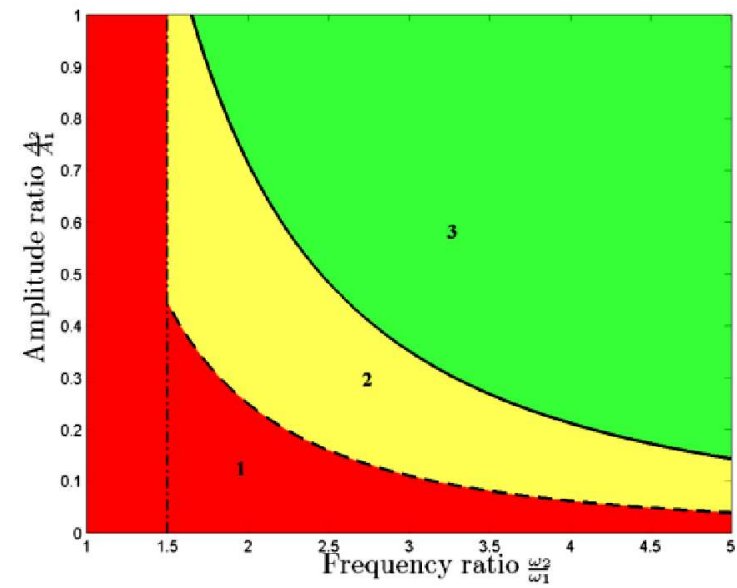

Fig. 5. Summary of the EMD ranges for harmonics separation: (1) impossible decomposition for very close frequency harmonics and small amplitude ratio; (2) decomposition requires several sifting iterations for close frequency harmonics; (3) single iteration separation for distant frequency harmonics and large amplitude ratio [11]

Further research is needed with the focus on the using of the selected sifting levels combinations (e.g. IMF2 and IMF3 band).

Mentioned combination can enable separation $T_{I}$ and $T_{\text {II }}$ signals as well as allow accurate estimation of vibration amplitude values of turbine components in the considered mechanical system.

\section{References}

1. C. J. Stander, P.S. Heyns, W. Schoombie, Using vibration monitoring for local fault detection on gears operating under fluctuating load conditions, Mechanical Systems and Signal Processing 166 (2002)

2. M. Zhao, J. Lin, X. Xu, Y. Lei, Tacholess envelope order analysis and its application to fault detection of rolling element bearings with varying speeds, Sensors 138 (2013)

3. H. Li, Y. Zhang, H. Zheng, Bearing fault detection and diagnosis based on order tracking and TeagerHuang Transform, Journal of Mechanical Science and Technology, 243 (2010)

4. R. Ricci, P. Pennacchi, Diagnostics of gear faults based on EMD and automatic selection of intrinsic mode functions, Mechanical Systems and Signal Processing (2011)

5. J. Dybała, R. Zimroz, Application of Empirical Mode Decomposition for impulsive signal extraction to detect bearing damage- industrial case study, Condition Monitoring of Machinery in Non-Stationary Operations, Springer Berlin Heidelberg (2012) 
6. P. Gaur, R.B. Pachori, H. Wang, G. Prasad, An empirical mode decomposition based filtering method for classification of motor- imagery EEG signals for enhancing brain- computer interface, Neural Networks (IJCNN), 2015 International Joint Conference on, IEE, 1-7 (2015)

7. Y. Yuan, C. Sun, M. Li, S.S. Choi, Q. Li, Determination of optimal supercapacitor-lead-acid battery energy storage capacity for smoothing wind power using empirical mode decomposition and neural network, Electric Power Systems Research, 127 (2015)

8. N.E. Huang, Z. Shen, S.R. Long, M.C. Wu, H.H. Shih, Q. Zheng, H.H. Liu, The empirical mode decomposition and the Hilbert spectrum for nonlinear and non-stationary time series analysis, Proceedings of the Royal Society of London A: Mathematical Physical and Engineering Sciences, 454 1971, The Royal Society (1998)

9. J. Dybała, R. Zimroz, Rolling bearing diagnosing method based on empirical mode decomposition of machine vibration signal, Applied Acoustics 77 (2014)

10. D. Yang, H. Li, Y. Hu, J. Zhao, H. Xiao, Y. Lan, Vibration condition monitoring system for wind turbine bearings based on noise suppression with multi- point data fusion, Renewable Energy 92 (2016)

11. M. Feldman, Analytical basics of the EMD: Two harmonics decomposition, Mechanical Systems and Signal Processing, 237 (2009) 